\title{
How to make a linear network code (strongly) secure
}

\author{
Kaoru Kurosawa $^{1} \cdot$ Hiroyuki Ohta ${ }^{1} \cdot$ Kenji Kakuta $^{1}$
}

Received: 6 April 2015 / Revised: 23 October 2015 / Accepted: 9 January 2016 /

Published online: 28 January 2016

(C) The Author(s) 2016. This article is published with open access at Springerlink.com

\begin{abstract}
A linear network code is called $k$-secure if it is secure even if an adversary eavesdrops at most $k$ edges. In this paper, we show an efficient deterministic construction algorithm of a linear transformation $T$ that transforms an (insecure) linear network code to a $k$-secure one for any $k$, and extend this algorithm to strong $k$-security for any $k$. Our algorithms run in polynomial time if $k$ is a constant, and these time complexities are explicitly presented. We also present a concrete size of $|\mathrm{F}|$ for strong $k$-security, where $\mathrm{F}$ is the underling finite field.
\end{abstract}

Keywords Information theory $\cdot$ Network code $\cdot$ Security

Mathematics Subject Classification 94A17

\section{Introduction}

The notion of network code was introduced by Ahlswede et al. [1]. Li et al. [11] proved that the source node $s$ can multicast $n$ field elements $\left(m_{1}, \ldots, m_{n}\right)$ to a set of sink nodes Sink $=\left\{t_{1}, \ldots, t_{q}\right\}$ by using a linear network code if $|\mathrm{F}| \geq \mid$ Sink $\mid$, where

$$
n=\min _{i} \max -\text { flow }\left(s, t_{i}\right)
$$

and $\mathrm{F}$ is a finite field such that $m_{i} \in \mathrm{F}$. (Fig. 1 shows an example of a linear network code.) Jaggi et al. [8] proposed a polynomial time algorithm which can construct a linear network code from any network instance $(G(\mathcal{V}, \mathcal{E}), s$, Sink, $n)$, where $G(\mathcal{V}, \mathcal{E})$ is the underlying network.

Communicated by M. Paterson.

A preliminary version of this paper appeared in [10].

$\triangle$ Kaoru Kurosawa

kurosawa@mx.ibaraki.ac.jp; kaoru.kurosawa.kk@vc.mx.ibaraki.ac.jp

1 Ibaraki University, 4-12-1 Nakanarusawa, Hitachi, Ibaraki 316-8511, Japan 


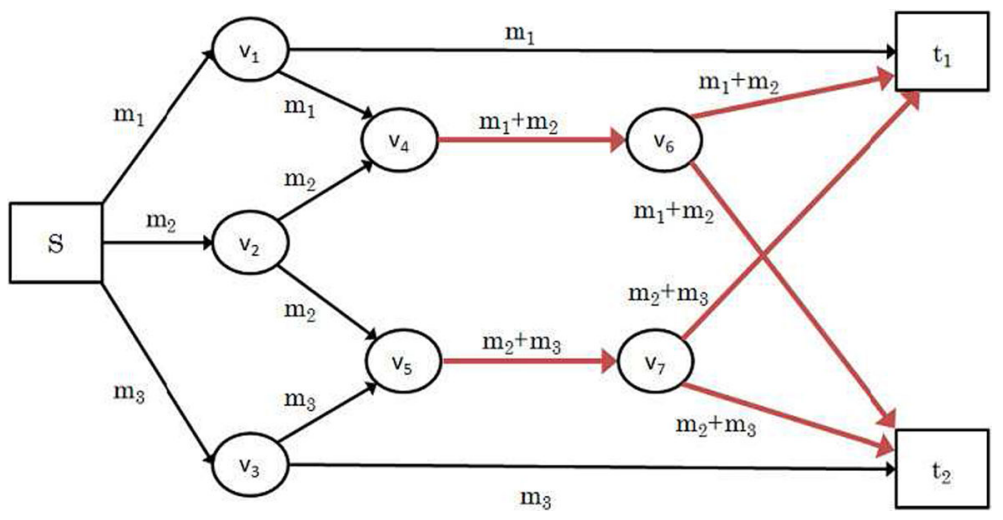

Fig. 1 Linear network coding scheme with $n=3$

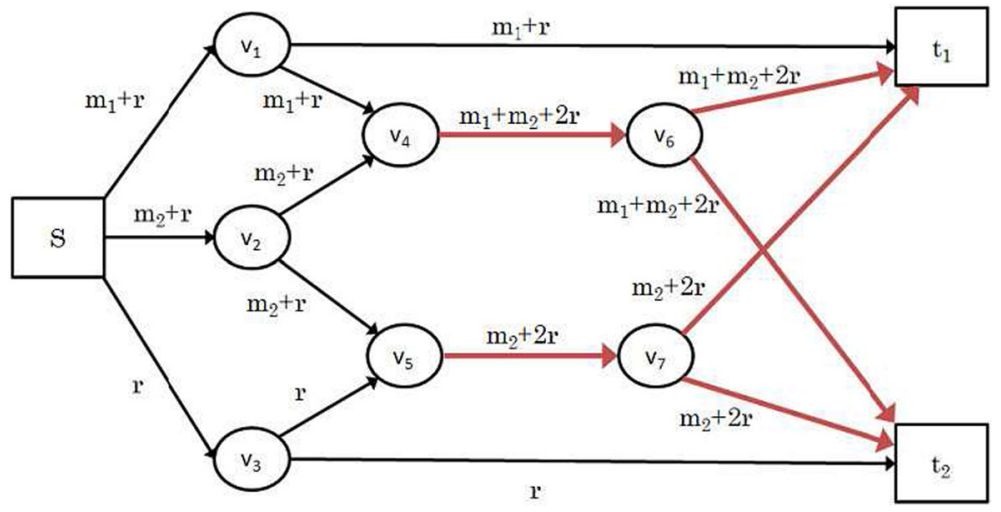

Fig. 2 1-Secure linear network coding scheme $(\bmod 3)$

Consider a model such that the source node $s$ multicasts $\left(m_{1}, \ldots, m_{n-k}, r_{1}, \ldots, r_{k}\right)$ instead of $\left(m_{1}, \ldots, m_{n}\right)$, where $r_{i}$ is chosen uniformly at random from the field $F$. We say that a linear network code is $k$-secure if an adversary learns no information on $\left(m_{1}, \ldots, m_{n-k}\right)$ even by eavesdropping at most $k$ edges.

Figure 2 shows a 1 -secure linear network code. For example, $d_{1}=m_{1}+r$ is transmitted on the edge $\left(s, v_{1}\right)$. It leaks no information on $m_{1}$ because the random element $r$ works as one-time pad.

Cai and Yeung [4] proved that there exists a linear transformation $T$ that makes any linear network code $k$-secure if $|\mathrm{F}|>\left(\begin{array}{c}|\mathcal{E}| \\ k\end{array}\right)$, where $\mathcal{E}$ is the set of edges. In fact, $T$ is an $n \times n$ nonsingular matrix.

The advantage of this method is that it does not require changing the underlying linear network code [6]. The source node $s$ only has to multicast $\left(\tilde{m}_{1}, \ldots, \tilde{m}_{n}\right)=$ $\left(m_{1}, \ldots, m_{n-k}, r_{1}, \ldots, r_{k}\right) \times T$.

Cai and Yeung, however, only showed the existence of $T$ based on a counting argument (see [4, Sect. V]). They did not show how to construct $T$ efficiently.

Harada and Yamamoto [7] extended the notion of $k$-security to strong $k$-security. Consider any $A \subset \mathcal{E}$ and any $B \subset\left\{m_{1}, \ldots, m_{n}\right\}$ such that $|A|=|B| \leq k$. Then a linear network code is called strongly $k$-secure if $A$ leaks no information on $\left\{m_{1}, \ldots, m_{n}\right\} \backslash B$. 


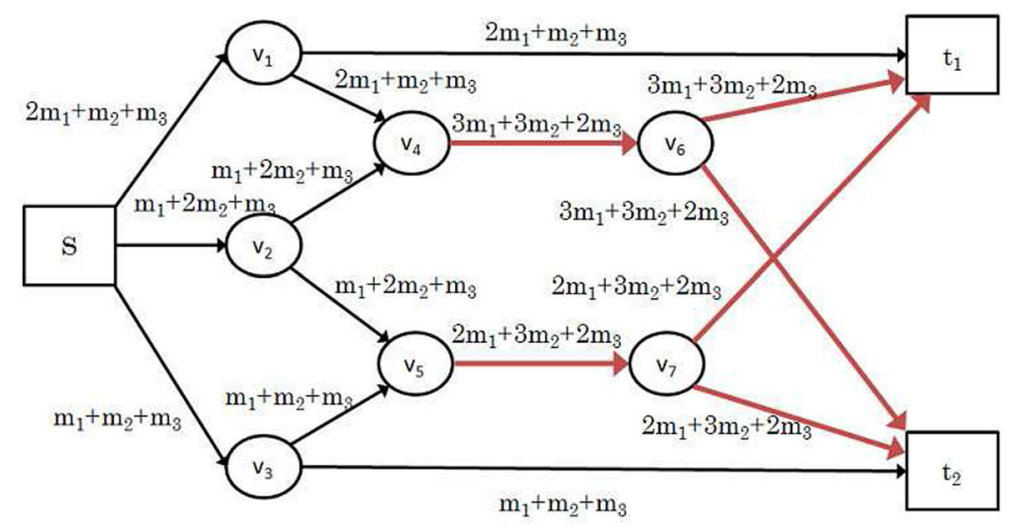

Fig. 3 Strongly 1 -secure linear network coding scheme $(\bmod 5)$

Figure 3 shows a strongly 1 -secure linear network code. For example, $d_{1}=2 m_{1}+m_{2}+m_{3}$ is transmitted on the edge $\left(s, v_{1}\right)$ and

- $d_{1}$ leaks no information on $\left(m_{1}, m_{2}\right)$ since $m_{3}$ works as one-time pad.

- $d_{1}$ leaks no information on $\left(m_{2}, m_{3}\right)$ since $2 m_{1}$ works as one-time pad.

- $d_{1}$ leaks no information on $\left(m_{1}, m_{3}\right)$ since $m_{2}$ works as one-time pad.

In this model, it is assumed that each $m_{i}$ is independently random.

Harada and Yamamoto proved that for sufficiently large $|\mathrm{F}|$, there exists a strongly $k$-secure linear network code for any network instance $(G(\mathcal{V}, \mathcal{E}), s$, Sink, $n)$ if $k<n$. However, they did not explicitly state the time complexity of their algorithm explicitly. In addition, they did not suggest a concrete size for $|F|$, leaving the derivation of a sufficient condition on $|F|$ as an open problem. (See "open problem" in Table 1 of [7]. They considered strong $k^{\prime}$-security with $k^{\prime} \leq k$, where $\ell$ is used instead of $k$ in [7].)

In this paper, we first show an efficient deterministic construction algorithm of a linear transformation $T$ that transforms an (insecure) linear network code to a $k$-secure one for any $1 \leq k<n$. We then extend this algorithm for strong $k$-security for any $1 \leq k<n$. Both of our algorithms run in polynomial time if $k$ is a constant.

We explicitly present the time complexities of our algorithms. We also present a concrete size of $|\mathrm{F}|$ for strong $k$-security, thereby solving the open problem of Harada and Yamamoto [7].

By applying our methods to Fig. 1, we can obtain the 1-secure linear network code shown in Fig. 2, the strongly 1-secure linear network code shown in Fig. 3 and the strongly 2-secure linear network code shown in Fig. 4.

\subsection{Related works}

Rouayheb et al. [5] proposed a direct method to construct a $k$-secure linear network code from a network instance $(G(\mathcal{V}, \mathcal{E}), s, \operatorname{Sink}, n)$. This method does not use a linear transformation $T$, and therefore requires changes to the underlying linear network code.

Bhattad and Narayanan [2] showed how to construct weakly secure linear network codes.

Silva and Kschischang [14,15] introduced the notion of universal $k$-secure codes and universal strongly $k$-secure codes. Kurihara et al. [9] improved the universal strongly $k$ secure codes of $[14,15]$. In these schemes $[9,14,15]$, a vector (instead of a field element) is 


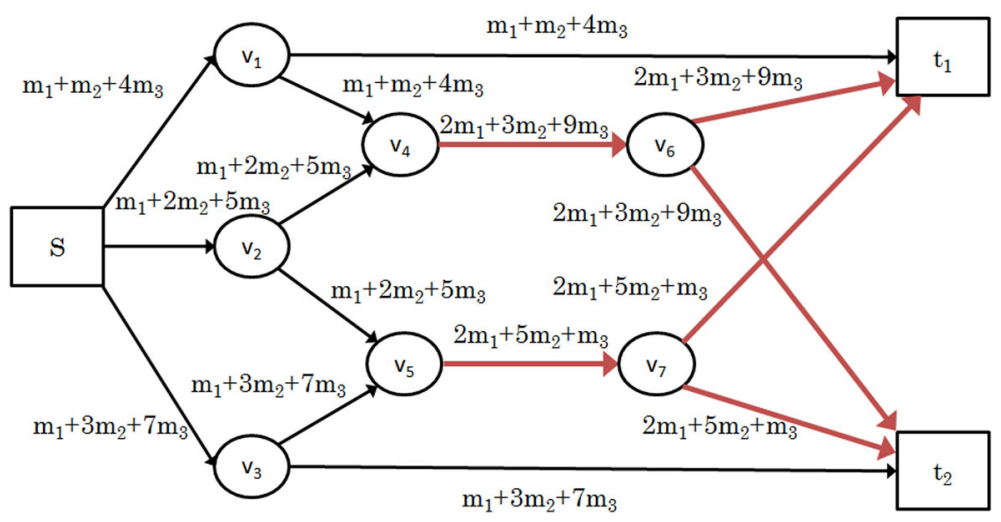

Fig. 4 Strongly 2-secure linear network coding scheme $(\bmod 11)$

transmitted over an edge. Because each element of the vector is transmitted over multiple time slots, it is assumed that the $k$ tapped edges are fixed during the transmission period. Shioji et al. [13] considered a stronger eavesdropping model where the adversaries possess the ability to re-select the tapping edges during the transmission. They then showed that the scheme in [15] is not secure under this eavesdropping model.

Matsumoto and Hayashi [12] considered a random linear precoder at the source node and proved that it is strongly secure and universal secure if we allow arbitrary small but nonzero mutual information on the transmission symbols to the eavesdropper. In their scheme, they showed that this mutual information is upper bounded by some small quantity.

Tang et al. [16] showed a probabilistic method to construct a linear transformation $T$ that transforms a linear network code to a $k$-secure one. (See "Time Complexity" of [16, p. 313].) However, they did not show the success probability and claimed (without proof) that the time complexity is $\left(\left(\begin{array}{c}|\mathcal{E}| \\ k\end{array}\right)\right)$.

\section{Preliminaries}

Let $H(\cdot)$ denote the Shannon entropy. For a tuple of random variables $\Lambda=\left(\tilde{a}_{1}, \ldots, \tilde{a}_{\ell}\right)$ and a subset $A=\left\{i_{1}, \ldots, i_{j}\right\} \subset\{1 \ldots \ell\}$, define

$$
\Lambda_{A}=\left(\tilde{a}_{i_{1}}, \ldots, \tilde{a}_{i_{\ell}}\right) .
$$

For a vector $\boldsymbol{x}=\left(x_{1}, \ldots, x_{N}\right)$, define

$$
\operatorname{support}(\boldsymbol{x})=\left\{i \mid x_{i} \neq 0\right\} .
$$

Let $w_{H}(\boldsymbol{x})$ denote its Hamming weight and $\boldsymbol{x}^{t}$ denote the transpose of $\boldsymbol{x}$. For a set $A$, let $|A|$ denote the cardinality of $A$.

For an $n \times \ell$ matrix $X$, define $X_{A}, X_{A, k}$ and $X_{A, B}$ as follows.

- For $A \subset\{1, \ldots, \ell\}$, let $X_{A}$ denote an $n \times|A|$ submatrix of $X$ such that the columns are restricted to $A$.

- For $k<n$, let $X_{A, k}$ denote a $k \times|A|$ submatrix of $X_{A}$ such that the rows are restricted to the last $k$ rows. Namely

$$
X_{A}=\left(\begin{array}{l}
Y \\
X_{A, k}
\end{array}\right)
$$


for some $Y$.

- For $B \subset\{1, \ldots, n\}, X_{A, B}$ denotes a $|B| \times|A|$ submatrix of $X_{A}$ such that the rows are restricted to $B$.

Definition 1 For an $n \times \ell$ matrix $X, \operatorname{Rank}_{p}(X)$ denotes the set of all $A \subset\{1, \ldots \ell\}$ such that

$$
|A|=\operatorname{rank}\left(X_{A}\right)=p .
$$

It is easy to see that the following lemma holds.

Lemma 1 Suppose that $T$ is a $n \times n$ nonsingularmatrix $T$. Then $\operatorname{Rank}_{p}(T \cdot X)=\operatorname{Rank}_{p}(X)$ for $p=1, \ldots, n-1$.

$\mathcal{I}_{\ell}$ denotes the $\ell \times \ell$ identity matrix. $\mathrm{F}$ denotes a finite field and, in particular, $\mathrm{F}_{p}$ denotes a finite field of order $p$.

\section{$3 k$-Secure linear network code}

\subsection{Linear network code}

We define a network instance by $(G(\mathcal{V}, \mathcal{E}), s$, Sink, $n)$ :

- $G(\mathcal{V}, \mathcal{E})$ is a directed acyclic network such that each edge $e \in \mathcal{E}$ has a unit capacity, i.e., each edge can transmit one field element per time unit. $G$ may include multiple parallel edges.

$-s \in \mathcal{V}$ is a source node.

- Sink $=\left\{t_{1}, \ldots, t_{q}\right\} \subset \mathcal{V}$ is a set of sink nodes.

- $n$ is defined as $n=\min _{i} \max$-flow $\left(s, t_{i}\right)$, where max-flow $\left(s, t_{i}\right)$ denotes the maximum flow from $s$ to $t_{i} \in$ Sink.

A linear network code for a network instance $(G(\mathcal{V}, \mathcal{E}), s$, Sink, $n)$ is defined by an $n \times|\mathcal{E}|$ linear network coding matrix $U$ such that

$$
\left(m_{1}, \ldots, m_{n}\right) \times U=\left(d_{1}, \ldots, d_{|\mathcal{E}|}\right),
$$

where $\left(m_{1}, \ldots, m_{n}\right)$ is the message that $s$ multicasts to Sink, and $d_{i}$ is the field element that is transmitted on an edge $e_{i} \in \mathcal{E}$. For example,

$$
U=\left(\begin{array}{l}
100110000101100 \\
010001100111111 \\
001000011010011
\end{array}\right)
$$

is the linear network coding matrix used in Fig. 1.

Formally we say that an $n \times|\mathcal{E}|$ matrix $U=\left(\boldsymbol{u}_{1}, \ldots, \boldsymbol{u}_{|\mathcal{E}|}\right)$ over $\mathrm{F}$ is a linear network coding matrix for a network instance $(G(\mathcal{V}, \mathcal{E}), s$, Sink, $n)$ if the following conditions are satisfied, where each $\boldsymbol{u}_{i}$ is indexed by an edge $e_{i} \in \mathcal{E}$.

1. If $e_{i} \in \mathcal{E}$ is an outgoing edge of a node $v(\neq s)$ and $v$ has incoming edges $e_{i_{1}}, \ldots, e_{i_{j}}$, then $\boldsymbol{u}_{i}$ is a linear combination of $\boldsymbol{u}_{i_{1}}, \ldots, \boldsymbol{u}_{i_{j}}$.

2. Let $\left\{e_{i_{1}}, \ldots, e_{i_{j}}\right\}$ be the set of incoming edges of a sink node $t_{i} \in$ Sink. Then $\operatorname{Rank}\left(\boldsymbol{u}_{i_{1}}, \ldots, \boldsymbol{u}_{i_{j}}\right)=n$ for each $t_{i} \in$ Sink. This condition guarantees that $t_{i}$ can reconstruct $\left(m_{1}, \ldots, m_{n}\right)$. 
Proposition 1 [8] There exists a polynomial time algorithm which can construct a linear network coding matrix $U$ from a network instance $(G, s, \operatorname{Sink}, n)$ if $|\mathrm{F}| \geq \mid$ Sink $\mid$.

Proposition 2 [6, Sect. 3] Suppose that $U$ is a linear network coding matrix for a network instance $(G, s, \operatorname{Sink}, n)$. Then for any $n \times n$ nonsingular matrix $T, T \times U$ is also a linear network coding matrix for $(G, s, \operatorname{Sink}, n)$.

Proposition 2 results from

$$
\left(m_{1}, \ldots, m_{n}\right) \times T \cdot U=\left(\tilde{m}_{1}, \ldots, \tilde{m}_{n}\right) \times U,
$$

where $\left(\tilde{m}_{1}, \ldots, \tilde{m}_{n}\right)=\left(m_{1}, \ldots, m_{n}\right) \times T$. Namely using $T \cdot U$ as a linear network coding matrix is equivalent to using $U$ as a linear network coding matrix such that $s$ multicasts $\left(\tilde{m}_{1}, \ldots, \tilde{m}_{n}\right)$. Each $t_{i}$ can reconstruct $\left(m_{1}, \ldots, m_{n}\right)$ because $T$ is nonsingular.

\section{$3.2 k$-Secure linear network code}

Consider the use of a linear network coding matrix $U$ such that

$$
\left(m_{1}, \ldots, m_{n-k}, r_{1}, \ldots, r_{k}\right) \times U=\left(d_{1}, \ldots, d_{|\mathcal{E}|}\right),
$$

where $\left(m_{1}, \ldots, m_{n-k}\right)$ is chosen according to some probability distribution, and each $r_{i}$ is independently and uniformly chosen from $\mathrm{F}$. Then, we say that $U$ is $k$-secure if any $k$ edges leak no information on $\left(m_{1}, \ldots, m_{n-k}\right)$.

More formally, let $\tilde{M}=\left(\tilde{m}_{1}, \ldots, \tilde{m}_{n-k}\right)$, where $\tilde{m}_{i}$ is the random variable induced by $m_{i}$ for $i=1, \ldots, n-k$, and let $\tilde{D}=\left(\tilde{d}_{1}, \ldots, \tilde{d}_{|\mathcal{E}|}\right)$, where $\tilde{d}_{j}$ is the random variable induced by $d_{j}$ for $j=1, \ldots,|\mathcal{E}|$. Then

Definition 2 A linear network coding matrix $U$ is $k$-secure if for any probability distribution on $\left(m_{1}, \ldots, m_{n-k}\right)$, it holds that

$$
H\left(\tilde{M} \mid \tilde{D}_{A}\right)=H(\tilde{M})
$$

for any $A \subset\{1 \ldots|\mathcal{E}|\}$ such that $|A|=k$.

Proposition 3 [3, Lemma 3.1] A linear network coding matrix $U=\left(u_{i, j}\right)$ is $k$-secure if and only if

$$
\operatorname{rank}\left(U_{A}\right)=\operatorname{rank}\left(U_{A, k}\right)
$$

for any $A \subseteq\{1, \ldots,|\mathcal{E}|\}$ such that $|A| \leq k$.

In particular, $U$ is 1 -secure if and only if $u_{n, i} \neq 0$ for all $i$.

Cai and Yeung proved the following proposition.

Proposition $4\left[4\right.$, Theorem 2] Suppose that $|\mathrm{F}|>\left(\begin{array}{c}|\mathcal{E}| \\ k\end{array}\right)$. Then for any $n \times|\mathcal{E}|$ linear network coding matrix $U$, there exists an $n \times n$ nonsingular matrix $T$ such that $V=T \times U$ is $k$-secure.

The advantage of this proposition is that no changes to $U$ are required. The source node $s$ only has to multicast $\left(\tilde{m}_{1}, \ldots, \tilde{m}_{n}\right)=\left(m_{1}, \ldots, m_{n-k}, r_{1}, \ldots, r_{k}\right) \times T$. (See the paragraph at the end of Sect. 3.1.)

Cai and Yeung, however, only showed the existence of $T$ based on a counting argument (see [4, Sect. V]). They did not show how to efficiently construct $T$. 


\section{Tools}

\subsection{Reduced coding matrix}

We say that a matrix $A=\left(\boldsymbol{a}_{1}, \ldots, \boldsymbol{a}_{h}\right)$ is pairwise column independent if each pair of columns $\left(\boldsymbol{a}_{i}, \boldsymbol{a}_{j}\right)$ are linearly independent.

For an $n \times|\mathcal{E}|$ linear coding matrix $U$, we say that an $n \times L$ matrix $\tilde{U}$ is a reduced coding matrix of $U$ if $\tilde{U}$ is a maximal submatrix of $U$ such that $\tilde{U}$ is pairwise column independent. This is formally define as follows.

Definition $3 \tilde{U}=\left(\tilde{\boldsymbol{u}}_{1}, \ldots, \tilde{\boldsymbol{u}}_{L}\right)$ is a reduced coding matrix of $U=\left(\boldsymbol{u}_{1}, \ldots, \boldsymbol{u}_{|\mathcal{E}|}\right)$ if

$-\left\{\tilde{\boldsymbol{u}}_{1}, \ldots, \tilde{\boldsymbol{u}}_{L}\right\} \subset\left\{\boldsymbol{u}_{1}, \ldots, \boldsymbol{u}_{|\mathcal{E}|}\right\}$,

- $\tilde{U}$ is pairwise column independent, and

- For any $\boldsymbol{u}_{i}$, there exists some $\tilde{\boldsymbol{u}}_{j}$ such that $\boldsymbol{u}_{i}=\beta \tilde{\boldsymbol{u}}_{j}$ for some $\beta \neq 0$.

We say that $L$ is the reduced size of $U$.

For example, the following matrix is a reduced coding matrix of $U$ given by Eq. (2)

$$
\tilde{U}=\left(\begin{array}{l}
10010 \\
01011 \\
00101
\end{array}\right)
$$

and $L=5$ is the reduced size of $U$.

Lemma 2 We can compute $(\tilde{U}, L)$ from $U=\left(\boldsymbol{u}_{1}, \ldots, \boldsymbol{u}_{|\mathcal{E}|}\right)$ in time $O\left(n|\mathcal{E}|^{2} \cdot \operatorname{poly}(\log |F|)\right)$.

Proof We can check if $\boldsymbol{u}_{i}$ and $\boldsymbol{u}_{j}$ are linearly independent in $O(n \cdot \operatorname{poly}(\log |F|))$ time. (Addition, subtraction, multiplication and devision in $\mathrm{F}$ takes $O($ poly $(\log |F|))$ times.) Therefore we can compute $(\tilde{U}, L)$ in $O\left(n|\mathcal{E}|^{2} \cdot \operatorname{poly}(\log |F|)\right)$ time.

It is easy to see that the following lemma holds.

Lemma 3 Suppose that $T$ is an $n \times n$ nonsingular matrix. Then, $T \cdot \tilde{U}$ is a reduced coding matrix of $T \cdot U$ if and only if $\tilde{U}$ is a reduced coding matrix of $U$.

It is clear that the following corollaries hold from Proposition 3.

Corollary 1 A linear network coding matrix $U$ is $k$-secure if and only if

$$
\operatorname{rank}\left(\tilde{U}_{A}\right)=\operatorname{rank}\left(\tilde{U}_{A, k}\right)
$$

for any $A \subset\{1, \ldots, L\}$ such that $|A| \leq k$.

Corollary 2 A linear network coding matrix $U$ is $k$-secure if and only if for $i=1, \ldots, k$, $\operatorname{rank}\left(\tilde{U}_{A, i}\right)=i$ for any $A_{i} \in \operatorname{Rank}_{i}(\tilde{U})$.

Corollary 3 A linear network coding matrix $U$ is 1-secure if and only if the last element of $\tilde{\boldsymbol{u}}_{i}$ is nonzero for each column vector $\tilde{\boldsymbol{u}}_{i}$ of $\tilde{U}$. 


\subsection{How to increase Hamming weight}

For two vectors $\boldsymbol{x}=\left(x_{1}, \ldots, x_{N}\right)$ and $\boldsymbol{y}=\left(y_{1}, \ldots, y_{N}\right)$, we present an algorithm MaxWeight $(\boldsymbol{x}, \boldsymbol{y})$ that finds $\alpha$ such that

$$
\operatorname{support}(\alpha \boldsymbol{x}+\boldsymbol{y})=\operatorname{support}(\boldsymbol{x}) \cup \operatorname{support}(\boldsymbol{y})
$$

in $O(N)$ time. To do so, we first show an algorithm that finds $\alpha \notin S$ in $O(|S|)$ time, where $S \subset \mathrm{F}$.

\section{Procedure: Outside $(S)$.}

Let the elements of $\mathrm{F}$ be $a_{0}, a_{1}, \ldots$.

1. Set $c(0)=\cdots=c(|S|)=0$.

2. For each $a \in S$, do:

3. If $a=a_{i}$ for some $i \leq|S|$, then set $c(i)=1$.

4. Let $i_{0}$ be the least $i$ such that $c(i)=0$. Return $a_{i_{0}}$ as $\alpha$.

For example,

- If $S=\left\{a_{0}, a_{1}, a_{2}\right\}$, then $c(0)=c(1)=c(2)=1$ and $c(3)=0$. Hence the above procedure returns $\alpha=a_{3}$.

- If $S=\left\{a_{0}, a_{1}, a_{4}\right\}$, then $c(0)=c(1)=1$ and $c(2)=c(3)=0$. Hence the above procedure returns $\alpha=a_{2}$.

It is easy to prove the following lemma.

Lemma 4 If $|\mathrm{F}|>|S|$, then Outside $(S)$ returns $\alpha \in \mathrm{F}$ such that $\alpha \notin S$ in $O(|S|)$ time.

We present the procedure for MaxWeight $(\boldsymbol{x}, \boldsymbol{y})$ as follows.

\section{Procedure: MaxWeight $(x, y)$.}

Let $\boldsymbol{x}=\left(x_{1}, \ldots, x_{N}\right)$ and $\boldsymbol{y}=\left(y_{1}, \ldots, y_{N}\right)$.

1. Let $S_{0}=\left\{-y_{i} / x_{i} \mid x_{i} \neq 0\right\}$.

2. $\alpha \leftarrow$ Outside $\left(S_{0}\right)$.

3. Return $\alpha$.

Lemma 5 For two vectors $\boldsymbol{x}=\left(x_{1}, \ldots, x_{N}\right)$ and $\boldsymbol{y}=\left(y_{1}, \ldots, y_{N}\right)$, MaxWeight $(\boldsymbol{x}, \boldsymbol{y})$ returns $\alpha$ such that

$$
\operatorname{support}(\alpha \boldsymbol{x}+\boldsymbol{y})=\operatorname{support}(\boldsymbol{x}) \cup \operatorname{support}(\boldsymbol{y})
$$

in $O(N \cdot \operatorname{poly}(\log |F|))$ if $|\mathrm{F}|>N$ time.

Proof Suppose that $|\mathrm{F}|>N$. Then because $|\mathrm{F}|>N \geq\left|S_{0}\right|$, we have $\alpha \notin S_{0}$ at line 2 of the above procedure according to Lemma 4 . This means that $\alpha x_{i}+y_{i} \neq 0$ if $x_{i} \neq 0$.

Hence if $\alpha x_{i}+y_{i}=0$, then $x_{i}=0$. This means $y_{i}=0$. Conversely if $x_{i}=y_{i}=0$, then $\alpha x_{i}+y_{i}=0$. Therefore $\alpha x_{i}+y_{i}=0$ if and only if $x_{i}=y_{i}=0$. In other words, $\alpha x_{i}+y_{i} \neq 0$ if and only if $x_{i} \neq 0$ or $y_{i} \neq 0$. Consequently we obtain Eq. (5).

Line 1 of the above procedure takes $O(N \cdot \operatorname{poly}(\log |F|))$ time because computing $y_{i} / x_{i}$ needs $O(\operatorname{poly}(\log |F|))$ time. At line 2, Outside $\left(S_{0}\right)$ runs in $O(N)$ time from Lemma 4. Therefore the algorithm runs in $O(N \cdot$ poly $(\log |F|))$ time.

For example, consider $\boldsymbol{x}=(1,1,1,0)$ and $\boldsymbol{y}=(0,4,2,3)$ over $\mathrm{F}_{5}$. Then $S_{0}=$ $\{0,-4,-2\}=\{0,1,3\}$ at line 1 . At line 2 , we obtain $\alpha=2 \notin S_{0}$. Then

$$
\alpha \boldsymbol{x}+\boldsymbol{y}=2 \times(1,1,1,0)+(0,4,2,3)=(2,1,4,3) .
$$

Thus Eq. (5) is satisfied. 


\subsection{Making a nonzero row}

Let $X$ and $Y$ be two $n \times \ell$ matrices. Let $\boldsymbol{x}_{i}$ denote the $i$ th row of $X$ and $\boldsymbol{y}_{i}$ denote the $i$ th row of $Y$ for $i=1, \ldots, n$. For $c \in\{1, \ldots, n\}$, we write

$$
Y \cong \text { except(c) } X
$$

if $\boldsymbol{y}_{i}=\boldsymbol{x}_{i}$ for all $i \neq c$. We also write

$$
Y \cong{ }_{\text {nonzero(c) }} X
$$

if $w_{H}\left(\boldsymbol{y}_{c}\right)=\ell$ and $\boldsymbol{y}_{i}=\boldsymbol{x}_{i}$ for all $i \neq c$. We present a deterministic polynomial time algorithm that outputs an $n \times n$ nonsingular matrix $T$ such that

$$
T \cdot X \cong_{\text {nonzero(c) }} X
$$

for $X$ that does not contain a column vector $(0, \ldots, 0)^{t}$ and

$$
T \cdot Y \cong \operatorname{except(c)} Y
$$

for any $Y$.

\section{Procedure: NonZeroRow $(\mathbf{X}, \mathrm{c})$}

Let $\boldsymbol{x}_{i}$ denote the $i$ th row of $X$ for $i=1, \ldots, n$.

1. $\boldsymbol{y}: \leftarrow \boldsymbol{x}_{c}$.

2. $\alpha_{c} \leftarrow 0$.

3. For $i=1, \ldots, n$, do:

4. If $i \neq c$, do:

5. $\quad \alpha_{i} \leftarrow \operatorname{MaxWeight}\left(\boldsymbol{x}_{i}, \boldsymbol{y}\right)$.

6. $\boldsymbol{y} \leftarrow \alpha_{i} \boldsymbol{x}_{i}+\boldsymbol{y}$.

7. Let $Q$ be an $n \times n$ matrix such that the $i$ th row is

$$
\mathbf{q}_{i}= \begin{cases}\left(\alpha_{1}, \ldots, \alpha_{n}\right) & \text { if } i=c \\ (0, \ldots, 0) & \text { if } i \neq c\end{cases}
$$

8. $T \leftarrow \mathcal{I}_{n}+Q$.

9. Return $T$.

Theorem 1 Let $X$ be an $n \times \ell$ matrix that does not contain a column vector $(0, \ldots, 0)^{t}$. Then the above algorithm outputs nonsingular matrix $T$ such that

$$
T \cdot X \cong_{\text {nonzero(c) }} X
$$

and

$$
T \cdot Y \cong{ }_{\operatorname{except}(\mathrm{c})} Y
$$

for any matrix $Y$ in $O(n \ell \cdot$ poly $(\log |F|))$ time if $|\mathrm{F}|>\ell$.

Proof Let $\boldsymbol{y}_{i}$ denote the $i$ th row of $T \cdot X$ for $i=1, \ldots, n$. Note that

$$
T \cdot X=(\mathcal{I}+Q) \cdot X=X+Q \cdot X .
$$

Therefore $\boldsymbol{y}_{i}=\boldsymbol{x}_{i}$ for $i \neq c$ and $\boldsymbol{y}_{c}$ is given as follows.

$$
\begin{aligned}
\boldsymbol{y}_{c} & =\boldsymbol{x}_{c}+\sum_{i=1}^{n} \alpha_{i} \boldsymbol{x}_{i} \\
& =\left(\ldots\left(\left(\boldsymbol{x}_{c}+\alpha_{1} \boldsymbol{x}_{1}\right)+\alpha_{2} \boldsymbol{x}_{2}\right)+\ldots\right)+\alpha_{n} \boldsymbol{x}_{n}
\end{aligned}
$$


Suppose that $|\mathrm{F}|>\ell$. Then, MaxWeight outputs $\alpha_{i}$ correctly at line 5 according to Lemma 5. Therefore

$$
w_{H}\left(\boldsymbol{y}_{c}\right)=\left|\operatorname{support}\left(\boldsymbol{y}_{c}\right)\right|=\left|\cup_{i=1}^{n} \operatorname{support}\left(\boldsymbol{x}_{i}\right)\right|=\ell
$$

because $X$ does not include $(0, \ldots, 0)^{t}$. Therefore

$$
T \cdot X \cong{ }_{\text {nonzero(c) }} X \text {. }
$$

By the same argument, it is easy to see that

$$
T \cdot Y \cong \text { except(c) } Y
$$

for any matrix $Y$.

Furthermore, it is clear that $T$ is nonsingular. Finally, line 5 takes $O(\ell \cdot \operatorname{poly}(\log |F|))$ time according to Lemma 5. Line 6 also takes $O(\ell \cdot \operatorname{poly}(\log |F|))$ time. Hence the algorithm runs in $O(n \ell \cdot \operatorname{poly}(\log |F|))$ time.

\section{Making a linear network coding matrix $k$-secure}

In this section, we propose the first efficient deterministic algorithm to compute a nonsingular matrix $T$ such that $T \times U$ is $k$-secure from a given linear network coding matrix $U$. Our algorithm runs in polynomial time if $k$ is a constant.

Furthermore, our algorithm succeeds if $|\mathrm{F}|>\left(\begin{array}{l}L \\ k\end{array}\right)$, where $L$ is the reduced size of $U$. Note that $|\mathrm{F}|>\left(\begin{array}{c}|\mathcal{E}| \\ k\end{array}\right)$ in Proposition 4 and $|\mathcal{E}| \geq L$. Therefore our sufficient condition on $|\mathrm{F}|$ is usually much smaller than that of Proposition 4.

Let $\tilde{U}$ be a reduced coding matrix of $U$.

\subsection{Making a linear network coding matrix 1-secure}

We begin by showing a polynomial time algorithm to compute an $n \times n$ nonsingular matrix $T$ such that $T \cdot U$ is 1 -secure. Our algorithm outputs $T$ such that the last row of $T \cdot \tilde{U}$ consists of nonzero elements. Then, $T \cdot U$ is 1 -secure according to Corollary 3 .

$\operatorname{Algorithm:~1-Secure}(\tilde{U}){ }^{1}$

1. $T \leftarrow \operatorname{NonZeroRow}(\tilde{U}, n)$.

2. Return $T$.

Theorem 2 The above algorithm outputs a nonsingular matrix $T$ such that the last row of $T \cdot \tilde{U}$ consists of nonzero elements in $O(n L \cdot$ poly $(\log |F|))$ time if $|\mathrm{F}|>L$, where $\tilde{U}$ is an $n \times L$ matrix.

Proof Follows from Theorem 1 . Note that no column vector of $\tilde{U}$ is $(0, \ldots, 0)^{t}$.

Corollary 4 We can construct a nonsingular matrix $T$ such that $T \cdot U$ is 1 -secure from any $n \times|\mathcal{E}|$ linear coding matrix $U$ in $O\left(n|\mathcal{E}|^{2} \cdot \operatorname{poly}(\log |F|)\right)$ time if $|\mathrm{F}|>L$, where $L$ is the reduced size of $U$.

1 A similar idea was used in [16, p. 309]. However, they did not show how to find $\alpha$ that appears in $\operatorname{MaxWeight}(\boldsymbol{x}, \boldsymbol{y})$ in polynomial time. They did not extend it to $k \geq 2$ like this paper, either. 
Proof Suppose that $|\mathrm{F}|>L$ and let $T$ be the output of 1-Secure $(\tilde{U})$. Note that $T \cdot \tilde{U}$ is a reduced coding matrix of $T \cdot U$ according to Lemma 3. Therefore $T \cdot U$ is 1 -secure according to Theorem 2 and Corollary 3.

We can compute $\tilde{U}$ from $U=\left(\boldsymbol{u}_{1}, \ldots, \boldsymbol{u}_{|\mathcal{E}|}\right)$ in $O\left(n|\mathcal{E}|^{2} \cdot \operatorname{poly}(\log |F|)\right)$ time according to Lemma 2. Note that $\max \left(n|\mathcal{E}|^{2}, n L\right)=n|\mathcal{E}|^{2}$. Therefore we can compute $T$ from $U$ in $O\left(n|\mathcal{E}|^{2} \cdot \operatorname{poly}(\log |F|)\right)$ time.

We now transform the insecure linear network code of Fig. 1 into a 1-secure one. By applying the above algorithm to $\tilde{U}$ of Eq. (4), we obtain. ${ }^{2}$

$$
T=\left(\begin{array}{lll}
1 & 0 & 0 \\
0 & 1 & 0 \\
1 & 1 & 1
\end{array}\right) \text { over } \mathrm{F}_{3} .
$$

The linear network coding matrix of Fig. 2 is $T \cdot U$, where $U$ is given by Eq. (2). Namely Fig. 2 is 1 -secure. ${ }^{3}$

\subsection{Making a linear network coding matrix 2-secure}

We now show a polynomial time algorithm to compute an $n \times n$ nonsingular matrix $T$ such that $V=T \cdot U$ is 2 -secure.

Lemma 6 For an $n \times L$ matrix $X$, suppose that

$$
\operatorname{rank}\left(X_{B, h-1}\right)=h-1
$$

for any $B \in \operatorname{Rank}_{h-1}(X)$. Then for any $A \in \operatorname{Rank}_{h}(X)$, we can find a nonzero vector a of length $h$ such that

$$
X_{A} \cdot \boldsymbol{a}=\left(\boldsymbol{c}, 0^{h-1}\right)^{t}
$$

for some c in $O\left(h^{3} \cdot\right.$ poly $\left.(\log |F|)\right)$ time. Furthermore,

$$
\operatorname{rank}\left(X_{A, h}\right)=h
$$

if and only if the last element of $c$ is nonzero.

Proof Suppose that $\operatorname{rank}\left(X_{B, h-1}\right)=h-1$ for any $B \in \operatorname{Rank}_{h-1}(X)$. Fix $A \subset\{1, \ldots, L\}$ such that $A \in \operatorname{Rank}_{h}(X)$ arbitrarily. Then an $h \times h$ matrix $X_{A, h}$ includes an $(h-1) \times(h-1)$ submatrix $X_{B, h-1}$ such that $\operatorname{rank}\left(X_{B, h-1}\right)=h-1$. Therefore $\operatorname{rank}\left(V_{A, h}\right)=h$ or $h-1$.

Let

$$
X_{A}=\left(\begin{array}{l}
Y_{1} \\
X_{A, h-1}
\end{array}\right)=\left(\begin{array}{l}
Y_{2} \\
X_{A, h}
\end{array}\right),
$$

where $X_{A, h-1}$ is an $(h-1) \times h$ submatrix and $X_{A, h}$ is an $h \times h$ submatrix. For a vector $\boldsymbol{a}$ of length $h$, we have

$$
X_{A} \cdot \boldsymbol{a}=\left(\begin{array}{l}
Y_{1} \cdot \boldsymbol{a} \\
X_{A, h-1} \cdot \boldsymbol{a}
\end{array}\right)=\left(\begin{array}{l}
Y_{2} \cdot \boldsymbol{a} \\
X_{A, h} \cdot \boldsymbol{a}
\end{array}\right) .
$$

\footnotetext{
2 We can often compute $T$ with smaller $|\mathrm{F}|$ than the sufficient condition stated in Corollary 4 (see Sect. 8.)

${ }^{3}$ We cannot use $F_{2}$ instead of $F_{3}$ because $m_{1}+m_{2}+2 r=m_{1}+m_{2} \bmod 2$. Hence Fig. 2 is not 1-secure if we use $F_{2}$.
} 
It is easy to see that $\operatorname{rank}\left(X_{A, h-1}\right)=h-1$ according to our assumption. Therefore we can find a nonzero vector $\boldsymbol{a}$ of length $h$ such that

$$
X_{A, h-1} \cdot \boldsymbol{a}=(0, \ldots, 0)^{t}
$$

in $O\left(h^{3} \cdot \operatorname{poly}(\log |F|)\right)$ time. We then have

$$
X_{A} \cdot \boldsymbol{a}=\left(\boldsymbol{c}, 0^{h-1}\right)^{t},
$$

where $\boldsymbol{c}=Y_{1} \cdot \boldsymbol{a}$. Let the last element of $\boldsymbol{c}$ be $c_{0}$. Hence

$$
X_{A, h} \cdot \boldsymbol{a}=\left(c_{0}, 0, \ldots, 0\right)^{t} .
$$

1. If $c_{0}=0$, then it is clear that $\operatorname{rank}\left(X_{A, h}\right)=h-1$.

2. Suppose that $\operatorname{rank}\left(X_{A, h}\right)=h-1$. Then for some $B \subset A$ such that $|B|=h-1$, there exists a vector $\boldsymbol{b}$ of length $h-1$ such that

$$
X_{B, h} \cdot \boldsymbol{b}=X_{A, h} \cdot \boldsymbol{a}=\left(c_{0}, 0, \ldots, 0\right)^{t} .
$$

This means that

$$
X_{B, h-1} \cdot \boldsymbol{b}=(0, \ldots, 0)^{t} .
$$

However, $\operatorname{rank}\left(X_{B, h-1}\right)=h-1$ according to our assumption. Hence we have $\boldsymbol{b}=$ $(0, \ldots, 0)^{t}$. This means that $c_{0}=0$ according to Eq. (6).

Therefore $c_{0}=0$ if and only if $\operatorname{rank}\left(X_{A, h}\right)=h-1$. Hence $c_{0} \neq 0$ if and only if $\operatorname{rank}\left(X_{A, h}\right)=h$.

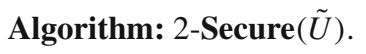

1. $T_{1} \leftarrow \operatorname{NonZeroRow}(\tilde{U}, n)$.

2. $V_{1} \leftarrow T_{1} \cdot \tilde{U}$.

3. For each $A_{i} \in \operatorname{Rank}_{2}(\tilde{U})$, find a nonzero vector $\boldsymbol{a}_{i}$ of length 2 such that

$$
\boldsymbol{y}_{i}=\left(V_{1}\right)_{A_{i}} \cdot \boldsymbol{a}_{i}=\left(\boldsymbol{c}_{i}, 0\right)^{t}
$$

for some $\boldsymbol{c}_{i}$.

4. Let $X=\left(\boldsymbol{y}_{1}, \boldsymbol{y}_{2}, \ldots\right)$.

5. $T_{2} \leftarrow \operatorname{NonZeroRow}(X, n-1)$.

6. $T \leftarrow T_{2} \cdot T_{1}$.

7. Return $T$.

As an example, suppose that $\tilde{U}$ is a $3 \times 2$ matrix such that $\operatorname{rank}(\tilde{U})=2$.

(1) At step 2, we obtain $V_{1}$ such that

$$
V_{1}=T_{1} \cdot \tilde{U}=\left(\begin{array}{ll}
* & * \\
* & * \\
\text { nonzero } & \text { nonzero }
\end{array}\right)
$$

because $T_{1}$ makes the last row of $\tilde{U}$ nonzero according to Theorem 1 .

(2) At step 3 , we find $\left(a_{1}, a_{2}\right) \neq(0,0)$ such that

$$
\boldsymbol{y}=V_{1} \cdot\left(\begin{array}{l}
a_{1} \\
a_{2}
\end{array}\right)=\left(\begin{array}{l}
c_{1} \\
c_{2} \\
0
\end{array}\right)
$$

for some $c_{1}, c_{2}$. 
(3) At step 5, we compute $T_{2}$ such that

$$
T_{2} \cdot \boldsymbol{y}=\left(\begin{array}{l}
c_{1} \\
\text { nonzero } \\
0
\end{array}\right)
$$

where $T_{2}$ makes the second element of $\boldsymbol{y}$ nonzero and does not change the other elements according to Theorem 1 .

Now let

$$
V_{2}=\left(\begin{array}{ll}
v_{11} & v_{12} \\
v_{21} & v_{22} \\
v_{31} & v_{32}
\end{array}\right)=T \cdot \tilde{U}=T_{2} \cdot T_{1} \cdot \tilde{U}
$$

Then

(a) First we have

$$
V_{2}=T_{2} \cdot\left(T_{1} \cdot \tilde{U}\right)=T_{2} \cdot\left(\begin{array}{ll}
* & * \\
* & * \\
\text { nonzero nonzero }
\end{array}\right)=\left(\begin{array}{ll}
* & * \\
*^{\prime} & *^{\prime} \\
\text { nonzero nonzero }
\end{array}\right) .
$$

because $T_{2}$ does not changes the third row of $T_{1} \cdot \tilde{U}$ according to Theorem 1 . Hence,

$$
v_{31} \neq 0 \text { and } v_{32} \neq 0
$$

(b) Second we have

$$
V_{2} \cdot\left(\begin{array}{l}
a_{1} \\
a_{2}
\end{array}\right)=T_{2} \cdot V_{1} \cdot\left(\begin{array}{l}
a_{1} \\
a_{2}
\end{array}\right)=T_{2} \cdot \boldsymbol{y}=\left(\begin{array}{l}
c_{1} \\
\text { nonzero } \\
0
\end{array}\right)
$$

Therefore

$$
\operatorname{rank}\left(\left(\begin{array}{ll}
v_{21} & v_{22} \\
v_{31} & v_{32}
\end{array}\right)\right)=2
$$

according to Lemma 6.

- Thus $V_{2}$ satisfies the condition of Corollary 2 from Eqs. (8) and (9).

- Furthermore, $V_{2}=T \cdot \tilde{U}$ is a reduced coding matrix of $T \cdot U$ from Lemma 3, where $U$ is the underlying linear network coding matrix.

- Consequently $T \cdot U$ is 2 -secure from Corollary 2.

Theorem 3 Let $\tilde{U}$ be an $n \times L$ matrix. If $|\mathrm{F}|>\left(\begin{array}{l}L \\ 2\end{array}\right)$, then the above algorithm outputs a nonsingular matrix $T$ in $O\left(n L^{2} \cdot\right.$ poly $\left.(\log |F|)\right)$ time as follows. Let $V_{2}=T \cdot \tilde{U}$. Then

(1) $\operatorname{rank}\left(\left(V_{2}\right)_{A, 1}\right)=1$ for any $A \in \operatorname{Rank}_{1}\left(V_{2}\right)$, and

(2) $\operatorname{rank}\left(\left(V_{2}\right)_{A, 2}\right)=2$ for any $A \in \operatorname{Rank}_{2}\left(V_{2}\right)$

Proof Suppose that $|\mathrm{F}|>\left(\begin{array}{l}L \\ 2\end{array}\right)$. At line 4 of 2-Secure $(\tilde{U})$, the number of columns of $X$ is equal to $\left(\begin{array}{l}L \\ 2\end{array}\right)$. Therefore, according to Theorem 1, NonZeroRow outputs $T_{2}$ correctly at line 5 . Namely, $T_{2}$ makes the $(n-1)$ th row of $X=\left(\boldsymbol{y}_{1}, \boldsymbol{y}_{2}, \ldots\right)$ nonzero and does not change the other rows. Also, NonZeroRow outputs $T_{1}$ correctly at line 1.

Note that $V_{1}=T_{1} \cdot \tilde{U}$ and

$$
V_{2}=T \cdot \tilde{U}=T_{2} \cdot T_{1} \cdot \tilde{U}=T_{2} \cdot V_{1} .
$$


Therefore

$$
\operatorname{Rank}_{p}\left(V_{2}\right)=\operatorname{Rank}_{p}\left(V_{1}\right)=\operatorname{Rank}_{p}(\tilde{U})
$$

for any $p$ according to Lemma 1 . We can thus write $A \in \operatorname{Rank}_{p}$ instead of $A \in \operatorname{Rank}_{p}(\tilde{U})$.

(a) The last row of $V_{1}=T_{1} \cdot \tilde{U}$ consists of nonzero elements according to Theorem 1 . This means that $\operatorname{rank}\left(\left(V_{1}\right)_{A, 1}\right)=1$ for any $A \in \operatorname{Rank}_{1}$.

(b) The last row of $V_{2}=T_{2} \cdot V_{1}$ also consists of nonzero elements because $T_{2}$ does not change the last row of $V_{1}$ according to Theorem 1 . Therefore $\operatorname{rank}\left(\left(V_{2}\right)_{A, 1}\right)=1$ for any $A \in$ Rank $_{1}$.

(c) Consider $A_{i} \in$ Rank $_{2}$ at step 3. From Lemma 6 and (a), we can find a nonzero vector $\boldsymbol{a}_{i}$ that satisfies Eq. (7). Then we have

$$
\begin{aligned}
\left(V_{2}\right)_{A_{i}} \cdot \boldsymbol{a}_{i} & =\left(T_{2} \cdot V_{1}\right)_{A_{i}} \cdot \boldsymbol{a}_{i} \\
& =T_{2} \cdot\left(\left(V_{1}\right)_{A_{i}} \cdot \boldsymbol{a}_{i}\right) \\
& =T_{2} \cdot \boldsymbol{y}_{i} \\
& =T_{2} \cdot\left(\boldsymbol{c}_{i}, 0\right)^{t} \\
& =\left(\boldsymbol{c}_{i}^{\prime}, 0\right)^{t}
\end{aligned}
$$

for some $\boldsymbol{c}_{i}^{\prime}$. The last equality holds because $T_{2}$ does not change the last element of $\left(c_{i}, 0\right)^{t}$.

(d) The last element of $\boldsymbol{c}_{i}^{\prime}$ is nonzero because $T_{2}$ makes the $(n-1)$ th element of $\boldsymbol{y}_{i}=\left(\boldsymbol{c}_{i}^{\prime}, 0\right)^{t}$ nonzero. Thus we have $\operatorname{rank}\left(\left(V_{2}\right)_{A_{i}, 2}\right)=2$ for any $A_{i} \in$ Rank $_{2}$ from Lemma 6.

From (b) and (e), we see that (1) and (2) of this theorem hold.

It is clear that $T$ is nonsingular. Finally the most time consuming part is line 5 . The number of columns of $X$ is $O\left(L^{2}\right)$. Hence line 5 runs in $O\left(n L^{2} \cdot \operatorname{poly}(\log |F|)\right)$ time according to Theorem 1. Therefore the algorithm runs in $O\left(n L^{2} \cdot\right.$ poly $\left.(\log |F|)\right)$ time.

Corollary 5 We can construct a nonsingular matrix $T$ such that $T \cdot U$ is 2-secure from any $n \times|\mathcal{E}|$ linear coding matrix $U$ in $O\left(n|\mathcal{E}|^{2} \cdot\right.$ poly $\left.(\log |F|)\right)$ time if $|\mathrm{F}|>\left(\begin{array}{c}L \\ 2\end{array}\right)$, where $L$ is the reduced size of $U$.

Proof Suppose that $|\mathrm{F}|>\left(\begin{array}{l}L \\ 2\end{array}\right)$ and let $T$ be the output of 2-Secure $(\tilde{U})$. Then $T \cdot \tilde{U}$ is a reduced coding matrix of $T \cdot U$ according to Lemma 3. Therefore, $T \cdot U$ is 2 -secure according to Theorem 2 and Corollary 2.

According to Lemma 2, we can compute $\tilde{U}$ from $U=\left(\boldsymbol{u}_{1}, \ldots, \boldsymbol{u}_{|\mathcal{E}|}\right)$ in $O\left(n|\mathcal{E}|^{2}\right.$. poly $(\log |F|))$ time. Note that $\max \left(n|\mathcal{E}|^{2}, n L^{2}\right)=n|\mathcal{E}|^{2}$. Therefore we can compute $T$ in $O\left(n|\mathcal{E}|^{2} \cdot\right.$ poly $\left.(\log |F|)\right)$ time from $U$.

\subsection{Making a linear network coding matrix $k$-secure}

Finally we show an efficient deterministic algorithm to compute an $n \times n$ nonsingular matrix $T$ such that $V=T \cdot U$ is $k$-secure for $3 \leq k<n$.

Algorithm: $k$-Secure $(\tilde{U})$.

1. $T_{1} \leftarrow \operatorname{NonZeroRow}(\tilde{U}, n)$.

2. $V_{1} \leftarrow T_{1} \cdot \tilde{U}$.

3. For $h=2, \ldots, k$, do:

4. For each $A_{i} \in \operatorname{Rank}_{h}(\tilde{U})$, find a nonzero vector $\boldsymbol{a}_{i}$ of length $h$ such that

$$
\boldsymbol{y}_{i}=\left(V_{h-1}\right)_{A_{i}} \cdot \boldsymbol{a}_{i}=\left(\boldsymbol{c}_{i}, 0^{h-1}\right)^{t}
$$




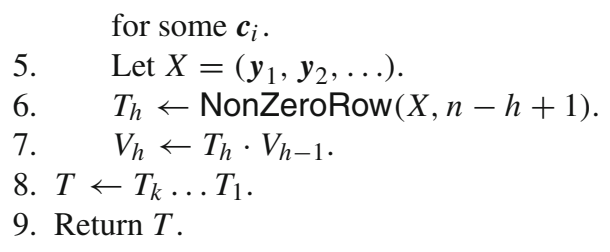

Theorem 4 Let $\tilde{U}$ be an $n \times L$ matrix. If $|\mathrm{F}|>\left(\begin{array}{l}L \\ k\end{array}\right)$, then the above algorithm outputs a nonsingular matrix $T$ in $O\left(k n L^{k} \cdot\right.$ poly $\left.(\log |F|)\right)$ time as follows. Let $V_{k}=T \cdot \tilde{U}$. Then

$$
\operatorname{rank}\left(\left(V_{k}\right)_{A, h}\right)=h
$$

for any $A \in \operatorname{Rank}_{h}\left(V_{k}\right)$ for $h=1, \ldots, k$.

Proof Suppose that $|\mathrm{F}|>\left(\begin{array}{l}L \\ k\end{array}\right)$. At line 5 of $k$-Secure $(\tilde{U})$, the number of columns of $X$ is at most $\left(\begin{array}{l}L \\ k\end{array}\right)$. Therefore from Theorem 1, NonZeroRow outputs $T_{h}$ correctly at line 6 . Furthermore NonZeroRow also outputs $T_{1}$ correctly at line 1 .

We say that an $n \times L$ matrix $V$ is bottom $h$ full independent if $\operatorname{rank}\left(V_{A, i}\right)=i$ for any $A \in \operatorname{Rank}_{i}(V)$ for $i=1, \ldots, h$. Suppose that $T$ is an $n \times n$ nonsingular matrix. Then $T \cdot V$ is bottom $h$ full independent if $V$ is bottom $h$ full independent.

$V_{1}=T_{1} \cdot \tilde{U}$ is bottom 1 full independent from the property of $T_{1}$. Suppose that $V_{h}$ is bottom $h$ full independent. By applying the same argument as in the proof of Theorem 3, we can see that $V_{h+1}$ is bottom $h+1$ full independent. Therefore $V_{k}$ is bottom $k$ full independent by induction. Hence Eq. (11) holds for any $A \in \operatorname{Rank}_{h}\left(V_{k}\right)$ for $h=1, \ldots, k$.

It is clear that $T$ is nonsingular. Finally the most time consuming part is line 6 . The number of columns of $X$ is $O\left(L^{k}\right)$. Hence line 6 runs in time $O\left(n L^{k} \cdot \operatorname{poly}(\log |F|)\right)$ from Theorem 1. Therefore the algorithm runs in in time $O\left(k n L^{k} \cdot \operatorname{poly}(\log |F|)\right)$.

Corollary 6 We can construct a nonsingular matrix $T$ such that $T \cdot U$ is $k$-secure from any $n \times|\mathcal{E}|$ linear coding matrix $U$ in time $O\left(\left(n|\mathcal{E}|^{2}+k n L^{k}\right) \cdot \operatorname{poly}(\log |F|)\right)$ if $|\mathrm{F}|>\left(\begin{array}{l}L \\ k\end{array}\right)$, where $L$ is the reduced size of $U$.

Proof Similar to the proof of Corollary 5.

\section{Strongly $k$-secure network coding scheme}

In Eq. (1), suppose that each $m_{i}$ is independently and uniformly distributed over F. Let $\tilde{m}_{i}$ denote this random variable induced by $m_{i}$ for $i=1, \ldots, n$, and let $\tilde{d}_{j}$ denote the random variable induced by $d_{j}$ for $j=1, \ldots,|\mathcal{E}|$. For $B \subset\{1, \ldots, n\}$, let $\bar{B}=\{1, \ldots, n\} \backslash B$. Then we define a strongly $k$-secure network coding matrix as follows.

Definition 4 A linear network coding matrix $U$ is strongly $k$-secure if

$$
H\left(\left(\tilde{m}_{1}, \ldots, \tilde{m}_{n}\right)_{\bar{B}} \mid\left(\tilde{d}_{1}, \ldots, \tilde{d}_{|\mathcal{E}|}\right)_{A}\right)=H\left(\left(\tilde{m}_{1}, \ldots, \tilde{m}_{n}\right)_{\bar{B}}\right)
$$

for any $A \subset\{1, \ldots|\mathcal{E}|\}$ and any $B \subset\{1 \ldots n\}$ such that $|A|=|B| \leq k$.

Harada and Yamamoto [7] proved the following proposition.

Proposition 5 [7, Theorem 3] For sufficiently large $|\mathrm{F}|$, there exists a strongly $k$-secure linear network coding matrix for any network instance $(G, s, \operatorname{Sink}, n)$ if $k<n$. 
However, they did not show the time complexity of their algorithm explicitly. They did not present a concrete size of $|\mathrm{F}|$ either. Indeed, they left it as an open problem to derive a sufficient condition on $|\mathrm{F}|$. (See "open problem" in Table 1 of [7]. They considered strong $k^{\prime}$-security with $k^{\prime} \leq k$, where $\ell$ is used instead of $k$ in [7].)

\subsection{Necessary and sufficient condition}

We can generalize Proposition 3 to strong $k$-security as follows.

Lemma 7 Consider $A \subset\{1, \ldots|\mathcal{E}|\}$ and $B \subset\{1 \ldots n\}$ such that $|A|=|B|$. Then Eq. (12) holds if and only if

$$
\operatorname{rank}\left(U_{A}\right)=\operatorname{rank}\left(U_{A, B}\right) .
$$

The proof is similar to that of Proposition 3 [3, Lemma 3.1].

Proof Without loss of generality, let $A=B=\{1, \ldots, k\}$. Then we have

$$
\begin{aligned}
\left(d_{1}, \ldots, d_{k}\right) & =\left(m_{1}, \ldots, m_{n}\right) \cdot U_{A} \\
& =\left(m_{1}, \ldots, m_{k}\right) \cdot U_{A, B}+\left(m_{k+1}, \ldots, m_{n}\right) \cdot U_{A, \bar{B}} \\
& =\left(r_{1}, \ldots, r_{k}\right)+\left(s_{k+1}, \ldots, s_{n}\right)
\end{aligned}
$$

where

$$
\begin{aligned}
\left(r_{1}, \ldots, r_{k}\right) & =\left(m_{1}, \ldots, m_{k}\right) \cdot U_{A, B} \\
\left(s_{k+1}, \ldots, s_{n}\right) & =\left(m_{k+1}, \ldots, m_{n}\right) \cdot U_{A, \bar{B}}
\end{aligned}
$$

Let $L_{0}$ be the image space of $U_{A}$ and $L_{1}$ be the image space of $U_{A, B}$.

Case 1 Suppose that $\operatorname{rank}\left(U_{A, B}\right)=\operatorname{rank}\left(U_{A}\right)$. Then $L_{1}=L_{0}$. Since $\left(m_{1}, \ldots, m_{k}\right)$ is a random vector, $\left(r_{1}, \ldots, r_{k}\right)$ is uniformly distributed over $L_{1}=L_{0}$. Therefore $\left(r_{1}, \ldots, r_{k}\right)$ works as one-time pad to mask $\left(s_{k+1}, \ldots, s_{n}\right)$. Hence Eq. (12) holds because one-time pad implies perfect secrecy.

Case 2 Suppose that $\operatorname{rank}\left(U_{A, B}\right)<\operatorname{rank}\left(U_{A}\right)$. Then $L_{1} \subset L_{0}$ and there exists some $\boldsymbol{y} \in L \backslash L_{1}$. Let $\tilde{r}_{i}$ denote the random variable induced by $r_{i}$ for $i=1, \ldots, k$, and $\tilde{s}_{i}$ denote the random variable induced by $s_{i}$ for $i=k+1, \ldots, n$. Then we have

$$
\begin{aligned}
& \operatorname{Pr}\left(\left(\tilde{d}_{1}, \ldots, \tilde{d}_{k}\right)=\boldsymbol{y} \mid\left(\tilde{s}_{1}, \ldots, \tilde{s}_{k}\right)=(0, \ldots, 0)\right) \\
& \quad=\operatorname{Pr}\left(\left(\tilde{r}_{1}, \ldots, \tilde{r}_{k}\right)=\boldsymbol{y}\right) \\
& \quad=0
\end{aligned}
$$

On the other hand, $\operatorname{Pr}\left(\left(\tilde{d}_{1}, \ldots, \tilde{d}_{k}\right)=\boldsymbol{y}\right)>0$ because each $m_{i}$ is independently random. Therefore

$$
\operatorname{Pr}\left(\left(\tilde{d}_{1}, \ldots, \tilde{d}_{k}\right)=\boldsymbol{y} \mid\left(\tilde{s}_{1}, \ldots, \tilde{s}_{k}\right)=(0, \ldots, 0)\right) \neq \operatorname{Pr}\left(\left(\tilde{d}_{1}, \ldots, \tilde{d}_{k}\right)=\boldsymbol{y}\right)
$$

Hence $\left(\tilde{d}_{1}, \ldots, \tilde{d}_{k}\right)$ and $\left(\tilde{s}_{1}, \ldots, \tilde{s}_{k}\right)$ are not independent. This means that Eq. (12) does not hold.

Theorem 5 A linear network coding matrix $U=\left(u_{i, j}\right)$ is strongly $k$-secure if and only if

$$
\operatorname{rank}\left(U_{A}\right)=\operatorname{rank}\left(U_{A, B}\right)
$$

for any $A \subset\{1, \ldots,|\mathcal{E}|\}$ and any $B \subset\{1, \ldots, n\}$ such that $|A|=|B| \leq k$.

Proof From Lemma 7. 


\subsection{Relation to reduced coding matrix}

Corollary 7 A linear network coding matrix $U$ is strongly 1-secure if and only if each element of $\tilde{U}$ is nonzero, where $\tilde{U}$ be a reduced coding matrix of $U$.

Corollary 8 A linear network coding matrix $U$ is strongly $k$-secure if and only if

$$
\operatorname{rank}\left(\tilde{U}_{A}\right)=\operatorname{rank}\left(\tilde{U}_{A, B}\right)
$$

for any $A \in \operatorname{Rank}_{p}(\tilde{U})$ and any $B \subset\{1, \ldots, n\}$ such that $|B|=$ for $p=1, \ldots, k$, where $\tilde{U}$ be a reduced coding matrix of $U$.

\section{How to make a linear network coding matrix strongly 1-secure}

In this section, we show the first efficient deterministic algorithm which computes a nonsingular matrix $T$ such that $T \times U$ is strongly $k$-secure from a given linear network coding matrix $U$. In particular, it runs in polynomial time if $k$ is a constant.

Let $\tilde{U}$ be a reduced coding matrix of $U$ and let $L$ be the reduced size of $U$. Then our algorithm succeeds if

$$
|\mathrm{F}|>L+\sum_{i=1}^{k-1}\left(\begin{array}{c}
n-1 \\
i
\end{array}\right)\left(\begin{array}{c}
L \\
i+1
\end{array}\right)
$$

\subsection{How to make a linear network coding matrix strongly 1-secure}

We first show a polynomial time algorithm which computes an $n \times n$ nonsingular matrix $T$ such that $T \cdot U$ is strongly 1 -secure. In fact, our algorithm outputs $T$ such that each element of $T \cdot \tilde{U}$ is nonzero. Then $T \cdot U$ is strongly 1 -secure from Corollary 7.

Algorithm: Strongly 1-Secure $(\tilde{U})$.

1. $T_{0} \leftarrow \operatorname{NonZeroRow}(\tilde{U}, n)$.

2. $V \leftarrow T_{0} \cdot \tilde{U}$.

Let $\boldsymbol{x}_{i}$ be the $i$ th row of $V$.

3. For $i=1, \ldots, n-1$, do:

4. $\quad \beta_{i} \leftarrow \operatorname{MaxWeight}\left(\boldsymbol{x}_{n}, \boldsymbol{x}_{i}\right)$.

5. Let

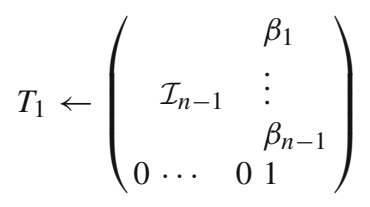

6. Return $T=T_{1} \times T_{0}$.

Theorem 6 Let $\tilde{U}$ be an $n \times L$ matrix. Then the above algorithm outputs a nonsingular matrix $T$ such that each element of $T \cdot \tilde{U}$ is nonzero in time $O(n L \cdot \operatorname{poly}(\log |F|))$ if $|\mathrm{F}|>L$. 
Proof Note that

$$
T \cdot \tilde{U}=T_{1} \cdot T_{0} \cdot \tilde{U}=T_{1} \cdot V=\left(\begin{array}{l}
\beta_{1} x_{n}+x_{1} \\
\vdots \\
\beta_{n-1} x_{n}+x_{n-1} \\
x_{n}
\end{array}\right)
$$

Suppose that $|\mathrm{F}|>L$. Then from Theorem 1, we have $w_{H}\left(\boldsymbol{x}_{n}\right)=L$ at line 2. Then from Lemma 5 , we have $w_{H}\left(\beta_{i} \boldsymbol{x}_{n}+\boldsymbol{x}_{i}\right)=L$ for $i=1, \ldots, n-1$. Therefore each element of $T \cdot \tilde{U}$ is nonzero.

Further it is clear that $T$ is nonsingular. Finally, line 1 takes time $O(n L \cdot \operatorname{pol} y(\log |F|))$ from Theorem 2, line 2 takes time $O(n L \cdot$ poly $(\log |F|))$, line 4 takes time $O(L \cdot \operatorname{poly}(\log |F|))$ from Lemma 5 and line 6 takes time $O\left(n^{2} \cdot \operatorname{poly}(\log |F|)\right)$. Hence the algorithm runs in time $O(n L \cdot \operatorname{poly}(\log |F|))$.

Corollary 9 We can construct a nonsingular matrix $T$ such that $T \cdot U$ is strongly 1-secure from any $n \times|\mathcal{E}|$ linear coding matrix $U$ in time $O\left(n|\mathcal{E}|^{2} \cdot\right.$ poly $\left.(\log |F|)\right)$ if $|\mathrm{F}|>L$, where $L$ is the reduced size of $U$.

Proof Similar to the proof of Corollary 5 where we use Corollary 7.

We transform the insecure linear network code of Fig. 1 into a strongly 1-secure one. By applying the above algorithm to $\tilde{U}$ of Eq. (4), we obtain ${ }^{4}$

$$
T=\left(\begin{array}{lll}
2 & 1 & 1 \\
1 & 2 & 1 \\
1 & 1 & 1
\end{array}\right) \text { over } \mathrm{F}_{5} .
$$

The linear network coding matrix of Fig. 3 is $T \cdot U$, where $U$ is given by Eq. (2). Namely Fig. 3 is strongly 1 -secure. ${ }^{5}$

\subsection{How to make a linear network coding matrix strongly 2-secure}

We next show a polynomial time algorithm which computes an $n \times n$ nonsingular matrix $T$ such that $V=T \cdot U$ is strongly 2 -secure.

Definition 5 For a subset $D \subset\{1, \ldots, n\}$ and an $n \times \ell$ matrix $E$, we say that a vector $\boldsymbol{y}=\left(y_{1}, \ldots, y_{n}\right)^{t}$ is a $D$-zero image of $E$ if (1) $\boldsymbol{y}=E \cdot \boldsymbol{a}$ for some nonzero vector $\boldsymbol{a}$ and (2) $y_{i}=0$ for each $i \in D$.

For example,

$$
\boldsymbol{y}=E \cdot \boldsymbol{a}=\left(0, y_{2}, \ldots, y_{n}\right)^{t}
$$

is a $\{1\}$-zero image of $E$.

Lemma 8 For a $p \times p$ matrix $X$, suppose that

$$
\operatorname{rank}\left(X_{B,\{1, \ldots, p-1\}}\right)=p-1
$$

\footnotetext{
${ }^{4}$ We can often compute $T$ with smaller $|\mathrm{F}|$ than the sufficient condition stated in Corollary 9. See Sect. 8.

5 We cannot use $F_{3}$ instead of $F_{5}$ because $3 m_{1}+3 m_{2}+2 m_{3}=m_{3}$ mod 3. Hence Fig. 3 is not strongly 1-secure if we use $\mathrm{F}_{3}$. 
for any $B \in \operatorname{Rank}_{p-1}(X)$. Then we can find a nonzero vector a of length $p$ such that

$$
X \cdot \boldsymbol{a}=\left(0^{p-1}, c\right)^{t}
$$

for some c in time $O\left(p^{3} \cdot\right.$ poly $\left.(\log |F|)\right)$. Further

$$
\operatorname{rank}(X)=p
$$

if and only if $c$ is nonzero.

Proof Similar to the proof of Lemma 6.

Algorithm: Strongly 2-Secure $(\tilde{U})$.

1. $T_{0} \leftarrow \operatorname{NonZeroRow}(\tilde{U}, 1)$.

2. $V_{0} \leftarrow T_{0} \cdot \tilde{U}$.

3. For $h=1, \ldots, n-1$, do:

4. For each $A_{i} \in \operatorname{Rank}_{2}(\tilde{U})$, find a $\{h\}$-zero image $\boldsymbol{y}_{i}$ of $\left(V_{h-1}\right)_{A_{i}}$.

5. $\quad W_{h} \leftarrow\left(\boldsymbol{y}_{1}, \boldsymbol{y}_{2}, \ldots\right)$.

6. $T_{h} \leftarrow \operatorname{NonZeroRow}\left(\left(V_{h-1}, W_{1}, \ldots, W_{h}\right), h+1\right)$.

7. $V_{h} \leftarrow T_{h} \cdot V_{h-1}$.

8. $\quad W_{1} \leftarrow T_{h} \cdot W_{1}$.

9.

10. $W_{h} \leftarrow T_{h} \cdot W_{h}$.

11. $T \leftarrow T_{n-1} \cdot \ldots \cdot T_{0}$.

12. Return $T$.

We illustrate how the algorithm proceeds for $n=3$.

1. At line 2:

$$
\begin{array}{|c|}
\hline V_{0}=T_{0} \tilde{U} \\
\hline \text { non-zero } \\
* * * \\
* * * \\
\hline
\end{array}
$$

2. In the first loop, at line 5,

\begin{tabular}{|c||c|}
\hline$V_{0}=T_{0} \tilde{U}$ & $W_{1}$ \\
\hline non-zero & $0, \ldots, 0$ \\
$* * *$ & $* * *$ \\
$* * *$ & $* * *$ \\
\hline
\end{tabular}

3. In the first loop, after line 8 ,

\begin{tabular}{|c||c|}
\hline$V_{1}=T_{1} T_{0} \tilde{U}$ & $W_{1}$ \\
\hline non-zero & $0, \ldots, 0$ \\
non-zero & non-zero \\
$* * *$ & $* * *$ \\
\hline
\end{tabular}

4. In the second loop, at line 5,

\begin{tabular}{|c|c||c|}
\hline$V_{1}=T_{1} T_{0} \tilde{U}$ & $W_{1}$ & $W_{2}$ \\
\hline non-zero & $0, \ldots, 0$ & $* * *$ \\
non-zero & non-zero & $0, \ldots, 0$ \\
$* * *$ & $* * *$ & $* * *$ \\
\hline
\end{tabular}


5. In the second loop, after line 10,

\begin{tabular}{|c|c||c|}
\hline$V_{2}=T_{2} T_{1} T_{0} \tilde{U}$ & $W_{1}$ & $W_{2}$ \\
\hline non-zero & $0, \ldots, 0$ & $* * *$ \\
non-zero & non-zero & $0, \ldots, 0$ \\
non-zero & non-zero & non-zero \\
\hline
\end{tabular}

We can see the following from the last table.

- Each element of $V_{2}$ is nonzero.

- From Lemma 8, for any $A_{i} \in \operatorname{Rank}_{2}\left(V_{2}\right)$,

$-\operatorname{rank}\left(\left(V_{2}\right)_{A_{i}, B}\right)=2$ for $B=\{1,2\}$ and $B=\{1,3\}$.

$-\operatorname{rank}\left(\left(V_{2}\right)_{A_{i}, B}\right)=2$ for $B=\{2,3\}$.

Hence $V=\left(T_{2} T_{1} T_{0}\right) U$ is strongly 2 -secure from Corollarys 7 and 8 .

Theorem 7 Let $\tilde{U}$ be an $n \times L$ matrix. Define

$$
\lambda=L+(n-1)\left(\begin{array}{l}
L \\
2
\end{array}\right) .
$$

If $|\mathrm{F}|>\lambda$. then the above algorithm outputs an $n \times n$ nonsingular matrix $T$ in time $O\left(n^{2} \lambda\right.$. poly $(\log |F|))$ such as fllows. Let $V_{n-1}=T \cdot \tilde{U}$. Then

(1) each element of $V_{n-1}$ is nonzero and

(2)

$$
\operatorname{rank}\left(\left(V_{n-1}\right)_{A, B}\right)=2
$$

for any $A \in \operatorname{Rank}_{2}\left(V_{n-1}\right)$ and any $B \subset\{1, \ldots, n\}$ such that $|B|=2$.

Proof Suppose that $|\mathrm{F}|>\lambda$. At line 6 , the number of columns of $\left(V_{h-1}, W_{1}, \ldots, W_{h}\right)$ is at most $\lambda$. Hence NonZeroRow outputs $T_{h}$ correctly from Theorem 1 . Namely $T_{h}$ makes the $(h+1)$ th row of $\left(V_{h-1}, W_{1}, \ldots, W_{h}\right)$ nonzero and does not change the other rows. Also NonZeroRow outputs $T_{0}$ correctly at line 1 , too.

For $1 \leq j \leq n-1$, it holds that

$$
\operatorname{Rank}_{2}\left(V_{j}\right)=\operatorname{Rank}_{2}(\tilde{U})
$$

from Lemma 1 because $V_{j}=T_{j} \ldots T_{0} \cdot \tilde{U}$. In this sense, we write $A \in$ Rank $_{2}$ instead of $A \in \operatorname{Rank}_{2}(\tilde{U})$.

(a) The first row of $V_{0}=T_{0} \cdot \tilde{U}$ consists of nonzero elements from Theorem 1.

(b) Suppose that

- the first $h$ rows of $V_{h-1}$ consists of nonzero elements, and

$-\operatorname{rank}\left(\left(V_{h-1}\right)_{A, B}\right)=2$ for any $A \in$ Rank $_{2}$ and any $B \subset\{1, \ldots, h\}$ such that $|B|=2$.

Then we will show that

- the first $h+1$ rows of $V_{h}$ consists of nonzero elements, and

$-\operatorname{rank}\left(\left(V_{h}\right)_{A, B}\right)=2$ for any $A \in$ Rank $_{2}$ and any $B \subset\{1, \ldots, h+1\}$ such that $|B|=2$.

(c) $T_{h}$ makes the $(h+1)$ th row of $\left(V_{h-1}, W_{1}, \ldots, W_{h}\right)$ nonzero and does not change the other rows. Therefore the first $h+1$ rows of $V_{h}=T_{h} \cdot V_{h-1}$ consists of nonzero elements from our assumption. 
Since $T_{h}$ does not change the first $h$ rows, we have $\operatorname{rank}\left(\left(V_{h}\right)_{A, B}\right)=2$ for any $A \in$ Rank $_{2}$ and any $B \subset\{1, \ldots, h\}$ such that $|B|=2$ from our assumption.

Now consider $B=\{j, h+1\}$ such that $j \in\{1, \ldots, h\}$.

- Each column vector $\boldsymbol{y}_{i}$ of $W_{h}$ is a $\{h\}$-image of $\left(V_{h-1}\right)_{A_{i}}$, where $A_{i} \in$ Rank $_{2}$. Therefore

$$
\boldsymbol{y}_{i}=\left(\boldsymbol{y}_{i, 1} 0, y_{h+1, i}, \boldsymbol{y}_{i, 2}\right)^{t}=\left(V_{h-1}\right)_{A_{i}} \cdot \boldsymbol{a}_{i},
$$

for some nonzero vector $\boldsymbol{a}_{i}$, where

$$
\begin{aligned}
& \boldsymbol{y}_{i, 1}=\left(y_{1, i}, \ldots, y_{h-1, i}\right) \\
& \boldsymbol{y}_{i, 2}=\left(y_{h+2, i}, \ldots, y_{n, i}\right)
\end{aligned}
$$

Multiply $T_{h}$ to the both hand sides. Then we have

$$
\begin{aligned}
T_{h} \cdot \boldsymbol{y}_{i} & =\left(\boldsymbol{y}_{i, 1} 0, \text { nonzero, } \boldsymbol{y}_{i, 2}\right)^{t} \\
& =\left(T_{h} \cdot V_{h-1}\right)_{A_{i}} \cdot \boldsymbol{a}_{i} \\
& =\left(V_{h}\right)_{A_{i}} \cdot \boldsymbol{a}_{i}
\end{aligned}
$$

because $T_{h}$ only makes the $(h+1)$ th element of $\boldsymbol{y}_{i}$ nonzero. Therefore we have

$$
\left(V_{h}\right)_{A_{i}} \cdot \boldsymbol{a}_{i}=\left(\boldsymbol{y}_{i, 1} 0, \text { nonzero, } \boldsymbol{y}_{i, 2}\right)^{t} \text {. }
$$

Then from Lemma 8, we have $\operatorname{rank}\left(\left(V_{h}\right)_{A_{i}, B}\right)=2$ for $B=\{h, h+1\}$ and $A_{i} \in$ Rank $_{2}$.

- By applying the same argument to $W_{1}, \ldots, W_{h-1}$, we can see that $\operatorname{rank}\left(\left(V_{h}\right)_{A, B}\right)=2$ for any $A \in$ Rank $_{2}$ and $B=\{j, h+1\}$ such that $j \in\{1, \ldots, h-1\}$.

Therefore $\operatorname{rank}\left(\left(V_{h}\right)_{A, B}\right)=2$ for any $A \in$ Rank $_{2}$ and any $B \subset\{1, \ldots, h+1\}$ such that $|B|=2$.

Consequently (1) and (2) of this theorem hold by induction.

It is clear that $T$ is nonsingular. Finally, the most time consuming part is line 6. It takes time $O(n \lambda \cdot \operatorname{poly}(\log |F|))$ from Theorem 1. Hence the algorithm runs in time $O\left(n^{2} \lambda\right.$. poly $(\log |F|))$.

Corollary 10 We can construct a nonsingular matrix $T$ such that $T \cdot U$ is strongly 2-secure from any $n \times|\mathcal{E}|$ linear coding matrix $U$ in time $O\left(\left(n|\mathcal{E}|^{2}+n^{2} \lambda\right) \cdot \operatorname{poly}(\log |F|)\right)$ if $|\mathrm{F}|>\lambda$, where

$$
\lambda=L+(n-1)\left(\begin{array}{l}
L \\
2
\end{array}\right)
$$

and $L$ is the reduced size of $U$.

Proof Similar to the proof of Corollary 5, where we use Corollary 8.

We transform the insecure linear network code of Fig. 1 into a strongly 2-secure one. By applying the above algorithm to $\tilde{U}$ of Eq. (4), we obtain ${ }^{6}$

$$
T=\left(\begin{array}{lll}
1 & 1 & 1 \\
1 & 2 & 3 \\
4 & 5 & 7
\end{array}\right) \text { over } F_{11} .
$$

The linear network coding matrix of Fig. 4 is $T \cdot U$, where $U$ is given by Eq. (2). Namely Fig. 4 is strongly 2 -secure ${ }^{7}$

\footnotetext{
6 We can often compute $T$ with smaller $|\mathrm{F}|$ than the sufficient condition stated in Corollary 10. See Sect. 8.

7 We cannot use $F_{7}$ instead of $F_{11}$ because $m_{1}+3 m_{2}+7 m_{3}=m_{1}+3 m_{2} \bmod 7$. Hence Fig. 4 is not strongly 2 -secure if we use $F_{7}$.
} 


\subsection{How to make a linear network coding matrix strongly $\boldsymbol{k}$-secure}

We finally show an efficient algorithm which computes an $n \times n$ nonsingular matrix $T$ such that $V=T \cdot U$ is strongly $k$-secure for $3 \leq k<n$.

Algorithm: Strongly $k$-Secure $(\tilde{U})$.

1. $T_{0} \leftarrow \operatorname{NonZeroRow}(\tilde{U}, 1)$.

2. $V_{0} \leftarrow T_{0} \cdot \tilde{U}$ and $X_{0} \leftarrow V_{0}$.

3. For $h=1, \ldots, n-1$, do:

4. $\quad$ For each $D_{j} \subset\{1, \ldots, h\}$ such that $h \in D_{j}$ and $\left|D_{j}\right|<k$, do:

5. For each $A_{i} \in \operatorname{Rank}_{\left|D_{j}\right|+1}(\tilde{U})$, find a $D_{j}$-zero image $\boldsymbol{y}_{i}$ of $\left(V_{h-1}\right)_{A_{i}}$.

6. $\quad W_{D_{j}} \leftarrow\left(\boldsymbol{y}_{1}, \boldsymbol{y}_{2}, \ldots\right)$.

7. $T_{h} \leftarrow$ NonZeroRow $\left(\left(V_{h-1}, X_{h-1}, W_{D_{1}}, W_{D_{2}}, \ldots\right), h+1\right)$.

8. $\quad V_{h} \leftarrow T_{h} \cdot V_{h-1}$.

9. $X_{h} \leftarrow T_{h} \cdot\left(X_{h-1}, W_{D_{1}}, W_{D_{2}}, \ldots\right)$.

10. $T \leftarrow T_{n-1} \cdot \ldots \cdot T_{0}$.

11. Return $T$.

We illustrate how the algorithm proceeds for $k=3$ and $n=4$.

1. At line 2,

\begin{tabular}{|c|}
\hline$V_{0}=T_{0} \tilde{U}$ \\
\hline non-zero \\
$* * *$ \\
$* * *$ \\
$* * *$ \\
\hline
\end{tabular}

2. At the end of the 1st loop,

\begin{tabular}{|c||c|}
\hline$V_{1}=T_{1} T_{0} \tilde{U}$ & $W_{\{1\}}$ \\
\hline non-zero & $0, \ldots, 0$ \\
non-zero & non-zero \\
$* * *$ & $* * *$ \\
$* * *$ & $* * *$ \\
\hline
\end{tabular}

3. At the end of the 2nd loop,

\begin{tabular}{|c|c||c|c|}
\hline$V_{2}=T_{2} T_{1} T_{0} \tilde{U}$ & $W_{\{1\}}$ & $W_{\{2\}}$ & $W_{\{1,2\}}$ \\
\hline non-zero & $0, \ldots, 0$ & $* * *$ & $0, \ldots, 0$ \\
non-zero & non-zero & $0, \ldots, 0$ & $0, \ldots, 0$ \\
non-zero & non-zero & non-zero & non-zero \\
$* * *$ & $* * *$ & $* * *$ & $* * *$ \\
\hline
\end{tabular}

4. At the end of the 3rd loop,

\begin{tabular}{|c|c||c|c||c|c|c|}
\hline$V_{3}=T_{3} \ldots T_{0} \tilde{U}$ & $W_{\{1\}}$ & $W_{\{2\}}$ & $W_{\{1,2\}}$ & $W_{\{3\}}$ & $W_{\{1,3\}}$ & $W_{\{2,3\}}$ \\
\hline non-zero & $0, \ldots, 0$ & $* * *$ & $0, \ldots, 0$ & $* * *$ & $0 \ldots 0$ & $* * *$ \\
non-zero & non-zero & $0, \ldots, 0$ & $0, \ldots, 0$ & $* * *$ & $* * *$ & $0 \ldots 0$ \\
non-zero & non-zero & non-zero & non-zero & $0 \ldots 0$ & $0 \ldots 0$ & $0 \ldots 0$ \\
non-zero & non-zero & non-zero & non-zero & non-zero & non-zero & non-zero \\
\hline
\end{tabular}

We can see the following from the last table. 
- Each element of $V_{3}$ is nonzero.

- By applying Lemma 8 to the columns indexed by $W_{\{1\}}, W_{\{2\}}$ and $W_{\{3\}}$, we can see that $\operatorname{rank}\left(\left(V_{3}\right)_{A_{i}, B}\right)=2$ for any $A_{i} \in \operatorname{Rank}_{2}\left(V_{3}\right)$ and for any $B \subset\{1,2,3,4\}$ with $|B|=2$.

- By applying Lemma 8 to the columns indexed by $W_{\{1,2\}}, W_{\{1,3\}}$ and $W_{\{2,3\}}$, we can see that $\operatorname{rank}\left(\left(V_{3}\right)_{A_{i}, B}\right)=3$ for any $A_{i} \in \operatorname{Rank}_{3}\left(V_{3}\right)$ and for any $B \subset\{1,2,3,4\}$ with $|B|=3$.

Therefore $V=\left(T_{3} T_{2} T_{1} T_{0}\right) U$ is strongly 3 -secure from Corollarys 7 and 8 .

Theorem 8 Let $\tilde{U}$ be an $n \times L$ matrix. Define

$$
\lambda=L+\sum_{i=1}^{k-1}\left(\begin{array}{c}
n-1 \\
i
\end{array}\right)\left(\begin{array}{c}
L \\
i+1
\end{array}\right) .
$$

If $|\mathrm{F}|>\lambda$, Then the above algorithm outputs an $n \times n$ nonsingular matrix $T$ in time $O\left(n^{2} \lambda\right.$. poly $(\log |F|))$ such that

$$
\operatorname{rank}\left((T \cdot U)_{A, B}\right)=p
$$

for any $A \in \operatorname{Rank}_{p}(\tilde{U})$ and any $B \subset\{1, \ldots, n\}$ with $|B|=p$ for $p=1, \ldots, k$.

Proof Suppose that $|\mathrm{F}|>\lambda$. At line 7, the number of columns of the matrix which is the input to NonZeroRow is at most $\lambda$. Hence NonZeroRow outputs $T_{h}$ correctly from Theorem 1 . Further NonZeroRow outputs $T_{0}$ correctly at line 1 , too.

We say that an $n \times L$ matrix $V$ is top $(h, k)$ full independent if $\operatorname{rank}\left(V_{A, B}\right)=|B|$ for any $B \subset\{1, \ldots, h\}$ such that $|B| \leq k$ and any $A \in \operatorname{Rank}_{|B|}(V)$. Suppose that $T$ is an $n \times n$ nonsingular matrix. Then $T \cdot V$ is top $(h, k)$ full independent if $V$ is top $(h, k)$ full independent.

$V_{0}=T_{0} \cdot \tilde{U}$ is top $(1,1)$ full independent because the first row of $V_{0}$ consists of nonzero elements from the property of $T_{0}$. Suppose that $V_{h-1}$ is top $(h, k)$ full independent. By applying the same argument as in the proof of Theorem 7, we can see that $V_{h}$ is top $(h+1, k)$ full independent. Therefore $V_{n-1}$ is top $(n, k)$ full independent by induction. Hence Eq. (16) holds for any $A \in \operatorname{Rank}_{p}(\tilde{U})$ and any $B \subset\{1, \ldots, n\}$ with $|B|=p$ for $p=1, \ldots, k$.

Furthermore, it is clear that $T$ is nonsingular. Finally, the most time consuming part is line 7. It takes $O(n \lambda \cdot \operatorname{pol} y(\log |F|))$ time according to Theorem 1 . Hence, the algorithm runs in $O\left(n^{2} \lambda \cdot \operatorname{poly}(\log |F|)\right)$ time.

Corollary 11 We can construct a nonsingular matrix $T$ from any $n \times|\mathcal{E}|$ linear coding matrix $U$ such that $T \cdot U$ is strongly $k$-secure in $O\left(\left(n|\mathcal{E}|^{2}+n^{2} \lambda\right) \cdot \operatorname{poly}(\log |F|)\right)$ time if $|\mathrm{F}|>\lambda$, where

$$
\lambda=L+\sum_{i=1}^{k-1}\left(\begin{array}{c}
n-1 \\
i
\end{array}\right)\left(\begin{array}{c}
L \\
i+1
\end{array}\right) .
$$

Proof Similar to the proof of Corollary 5, where we use Corollary 8.

\section{Summary}

We have proposed an efficient deterministic construction algorithm of a linear transformation $T$ that transforms a linear network code to a $k$-secure one for any $1 \leq k<n$. We have also extended this algorithm to strong $k$-security for any $1 \leq k<n$. Our algorithms run in 
polynomial time if $k$ is a constant, and these time complexities are explicitly presented. We also have presented a concrete size of $|\mathrm{F}|$ for strong $k$-security,

The condition on $|F|$ in Lemma 5 is a sufficient condition. Therefore MaxWeight $(\boldsymbol{x}, \boldsymbol{y})$ succeeds with smaller $|\mathrm{F}|$ as long as $|\mathrm{F}|>\left|S_{0}\right|$. For example, if the Hamming weight of $\boldsymbol{y}$ is small, then $\left|S_{0}\right|$ is small. Hence $|\mathrm{F}|$ can be small. For the same reason, our construction algorithms for the transformation matrix $T$ may succeed with smaller $|F|$ than the sufficient condition on $|\mathrm{F}|$ that is stated in each theorem.

Further work should explore if there exists a network instance such that our sufficient condition on $|\mathrm{F}|$ is tight.

Open Access This article is distributed under the terms of the Creative Commons Attribution 4.0 International License (http://creativecommons.org/licenses/by/4.0/), which permits unrestricted use, distribution, and reproduction in any medium, provided you give appropriate credit to the original author(s) and the source, provide a link to the Creative Commons license, and indicate if changes were made.

\section{References}

1. Ahlswede R., Cai N., Li S.-Y.R., Yeung R.W.: Network information flow. IEEE Trans. Inf. Theory 46(4), 1204-1216 (2000).

2. Bhattad K., Narayanan K.R.: Weakly secure network coding. In: Proceedings of NetCod 2005, April (2005).

3. Cai N., Yeung R.W.: A security condition for multi-source linear network coding. In: Proceedings of IEEE ISIT, 24-29 June, pp. 561-565 (2007).

4. Cai N., Yeung R.W.: Secure network coding on a wiretap network. IEEE Trans. Inf. Theory 57(1), 424-435 (2011).

5. El Rouayheb S., Soljanin E., Sprintson A.: Secure network coding for wiretap networks of type II. IEEE Trans. Inf. Theory 58(3), 1361-1371 (2012).

6. Feldman J., Malkin T., Servedio R., Stein C.: On the capacity of secure network coding. In: 42nd Annual Allerton Conference on Communication, Control, and Computing (2004).

7. Harada K., Yamamoto H.: Strongly secure linear network coding. IEICE Trans. 91-A(10), 2720-2728 (2008).

8. Jaggi S., Sanders P., Chou P.A., Effros M., Egner S., Jain K., Tolhuizen L.M.G.M.: Polynomial time algorithms for multicast network code construction. IEEE Trans. Inf. Theory 51(6), 1973-1982 (2005).

9. Kurihara J., Uematsu T., Matsumoto R.: Explicit construction of universal strongly secure network coding via MRD codes. In: Proceedings of IEEE ISIT 2012, Cambridge, MA, pp. 1488-1492 (2012).

10. Kurosawa K., Ohta H., Kakuta K.: How to construct strongly secure network coding scheme. In: ICITS, pp. 1-17 (2013).

11. Li S.Y.R., Yeung R.W., Cai N.: Linear network coding. IEEE Trans. Inf. Theory 49(2), 371-381 (2003).

12. Matsumoto R., Hayashi M.: Universal Strongly Secure Network Coding with Dependent and Non-uniform Messages. (2012). arXiv:1111.4174.

13. Shioji E., Matsumoto R., Uyematsu T.: Vulnerability of MRD-code-based universal secure network coding against stronger eavesdroppers. IEICE Trans. 93-A(11), 2026-2033 (2010).

14. Silva D., Kschischang F.R.: Universal weakly secure network coding. In: Proceedings of IEEE ITW 2009, pp. 281-285 (2009).

15. Silva D., Kschischang F.R.: Universal secure network coding via rank-metric codes. IEEE Trans. Inf. Theory 57(2), 1124-1135 (2011).

16. Tang Z., Lim H.W., Wang H.: Revisiting a secret sharing approach to network codes. In: ProvSec, pp. 300-317 (2012). 